\title{
Nonlinear gas oscillations in pipes. Part 2. Experiment
}

\author{
By B. STURTEVANT \\ Graduate Aeronautical Laboratories \\ California Institute of Technology, Pasadena
}

(Received 28 March 1973)

Forced nonlinear acoustic oscillations near the resonant frequency of closed and open tubes are studied experimentally. In particular, the motion in tubes terminated with different orifice plates is studied, and comparison is made with second- and third-order theories of the motion which contain an adjustable endwall reflexion coefficient.

It is found that oscillations at resonance in an open tube exhibit remarkably large amplitudes despite the fact that in some cases shock waves are emitted from the open end. For oscillations at resonance in a closed tube, the effect of substituting an orifice plate for the solid end wall is to reduce the amplitude and thicken the compressive portion of the shock waves which occur under these conditions. In both the open-tube and closed-tube experiments the reflexion coefficients which are evaluated by fitting theory to experiment are found to increase with increasing amplitude, in agreement with the observations of previous investigators (Ingard \& Ising 1967). In fact, for the open end the same linear dependence upon amplitude is observed, but the constant of proportionality is different. Qualitative differences are observed between the reflexion coefficients of a given orifice at the open-end and the closed-end resonant frequencies; at the open-end frequency the reflexion from the given orifice is less ideal than at the closed-end frequency. The implications of reflexion coefficients dependent on the wave forms are discussed.

\section{Introduction}

In the first paper of this series (Jimenez 1973) $\dagger$ the problem of forced acoustic oscillations in a pipe was studied theoretically. The present paper reports an experimental investigation of the same problem. We examine the response of both open and closed tubes to near-resonant excitation by large amplitude oscillations of a piston at one end of the tube.

The motion excited near resonance in a closed tube has previously been subjected to extensive experimental investigation. In particular, the pioneering experiments of Lettau (1939) and, later, of Saenger \& Hudson (1960) rather completely exhibited the qualitative features of the motion, the most notable of which is the appearance of shock waves in the tube at resonance. The discovery that shock waves occur for sufficiently large excitations stimulated several early

$\dagger$ Denoted hereafter as I. 


\begin{tabular}{|c|c|c|c|c|}
\hline & $\underset{(\mathrm{mm})}{l_{\max }}$ & $\begin{array}{l}L_{\text {typ }} \\
(\mathrm{m})\end{array}$ & $p_{\max }$ & Driver \\
\hline Lettau (1939) & 34 & 12 & $0 \cdot 20$ & Motor cycle engine \\
\hline Saenger \& Hudson (1960) & $\mathbf{3} \cdot \mathbf{2}$ & 1.7 & $0 \cdot 28$ & Electrodynamic shaker \\
\hline Temkin (1968) & $2 \cdot 0$ & 1.5 & $0 \cdot 16$ & Electromagnetic vibrator \\
\hline Cruikshank (1972) & 1.8 & $1 \cdot 7$ & $0 \cdot 14$ & Electrodynamic shaker \\
\hline Present work & 49 & $3 \cdot 2$ & 0.82 & Motorcycle engine \\
\hline
\end{tabular}

TABLE 1. Parameters of experiments with closed tubes. $l_{\max }=$ maximum report amplitude of piston motion; $L_{\mathrm{typ}}=$ typical length of resonant tube; $p_{\max }=\left(\left(\tilde{p}-\widetilde{p}_{0}\right) / \tilde{p}_{0}\right)_{\max }=$ maximum reported shock amplitude/mean pressure.

theoretical treatments of the nonlinear problem (cf. references of I), culminating in the theory of Chester (1964), which correctly predicted the occurrence and behaviour of shocks in a band of frequencies about the acoustic resonant frequency of the closed tube $\varpi_{c l}=\pi a_{0} / L$.

The several experimental investigations of nonlinear resonance in closed tubes have differed considerably in detail. Table 1 summarizes the values of important parameters that characterize previous and present experimental arrangements. The present apparatus was designed (Sturtevant 1970) specifically to maximize the amplitude of the piston motion.

In the present series we have extended consideration of the behaviour near the closed-tube resonant frequency to problems in which only partial reflexion occurs at the passive end of the pipe, and specifically, to the case in which the end is capped with an orifice plate; in I, Chester's second-order theory for closed tubes was extended to include partial reflexion, while in the present paper experimental results are presented. After a discussion in $\$ 3$ of the relationship between theory and experiment, the theory of $I$ is used in $\$ 4.1$ to evaluate the effective reflexion coefficient $b$ at the passive end of a closed tube from measurements of pressure wave forms ( $p$ vs. $t$ at fixed location) and pressure amplitudes. This reflexion coefficient, determined under the assumption that the fluid is ideal, is of course a measure of boundary-layer dissipation in the actual, viscous fluid (air) used in the experiments. In $\$ 4.2$, the effect of inserting orifice plates at the passive end is considered and the reflexion coefficients of the orifices are determined for oscillations in the neighbourhood of $\varpi_{c l}$. Since orifices are very effective in damping the motion it turns out that the reflexion coefficients of all but the smallest orifices may be determined by comparing observed amplitudes with linear theory rather than with the full nonlinear theory. $f$

The analogous problem of the nonlinear response of an open tube excited by a piston oscillating at the acoustic resonant frequency $\varpi_{0 p}=\pi a_{0} / 2 L$ was also studied by Lettau (1939), but his findings on this important problem attracted little attention. The investigation reported in the present papers was, in fact, stimulated by our own observation in exploratory experiments with an open tube of unexpectedly large motions at resonance; the amplitude of the observed

$\dagger$ The amplitude is much less sensitive to nonlinear effects than are the details of the wave form itself; in all of the experiments reported in this paper the wave forms are highly distorted. 
pressure wave form was nearly as large as at resonance in a closed tube. Indeed, the motions induced in the present apparatus are so large that, though the wave form at the piston is continuous and smooth, the compressive portions of the wave steepen and break in less than one tube length, and shock waves are emitted from the open end. This remarkable behaviour was observed by Lettau (1939) at higher harmonic excitation frequencies. In I a third-order theory for this problem is developed. By showing that nonlinear effects first appear in this problem at only the third order, the theory provides the explanation of the large amplitudes observed. In $\$ 5.1$ of this paper the theory of $I$ is used to evaluate the reflexion coefficient of an open-ended tube, while in $\$ 5.2$ the effect of inserting orifice plates at the end on oscillations in the neighbourhood of $\varpi_{o p}$ is described. In the final sections the results are compared and discussed.

The combination of a nonlinear resonant tube and orifice plates constitutes a prototype model of more complicated systems involving the interaction of finite amplitude wave fields with arbitrary geometrical configurations. Examples of such systems arise in a variety of problems ranging from the sonic boom to jet-engine inlets to the exhaust systems of small high-performance internal combusion engines or even to unstable rocket motors. By representing the effects of orifices, filters, mufflers, absorbers, etc., in terms of a reflexion coefficient and an effective tube length, the theory and experimental technique developed in this work provide a simple procedure for evaluating the reflective properties of such acoustic elements when subjected to intense sound fields. In analogy with an established tool of acoustics, the system studied here could be termed a 'nonlinear impedance tube'.

Since the flow through the orifice for moderate to strong acoustic fields is separated, turbulent and unsteady, little analytical progress has been made towards predicting the reflexion coefficient of orifice plates. Therefore, at present such properties must be determined empirically, and this paper presents some quantitative information about their behaviour.

\section{Experimental apparatus}

The tube used in the present experiments (figure 1) is made of aluminium and is 3 in. $(76 \mathrm{~mm})$ I.D. with $6.4 \mathrm{~mm}$ thick walls. Its length $L$ can be varied from 0.9 to $4.7 \mathrm{~m}$ in $0.75 \mathrm{~m}$ increments. The piston mechanism is a J.A. Prestwick four-cycle single-cylinder motor cycle engine $(80 \mathrm{~mm}$, bore $100 \mathrm{~mm}$ stroke), the cylinder head of which has been replaced by the $3 \mathrm{in}$. diameter resonance tube. Though the amplitude $l$ of the piston motion is fixed in these experiments, the normalized amplitude $l / L$ can be varied by more than a factor of 5 . The piston is driven by a variable-speed (0-6000 r.p.m.) 15 h.p. d.c. electric motor. Thus, the difficult problem of sealing and lubricating a high-speed $(20 \mathrm{~m} / \mathrm{s})$ piston mechanism has been solved by using an existing design. The only modifications to the original engine were the installation of a flat-head piston and rebalancing of the flywheel. The entire mechanical system is mounted on a one-ton bed which is dynamically isolated from the laboratory floor by air springs. 


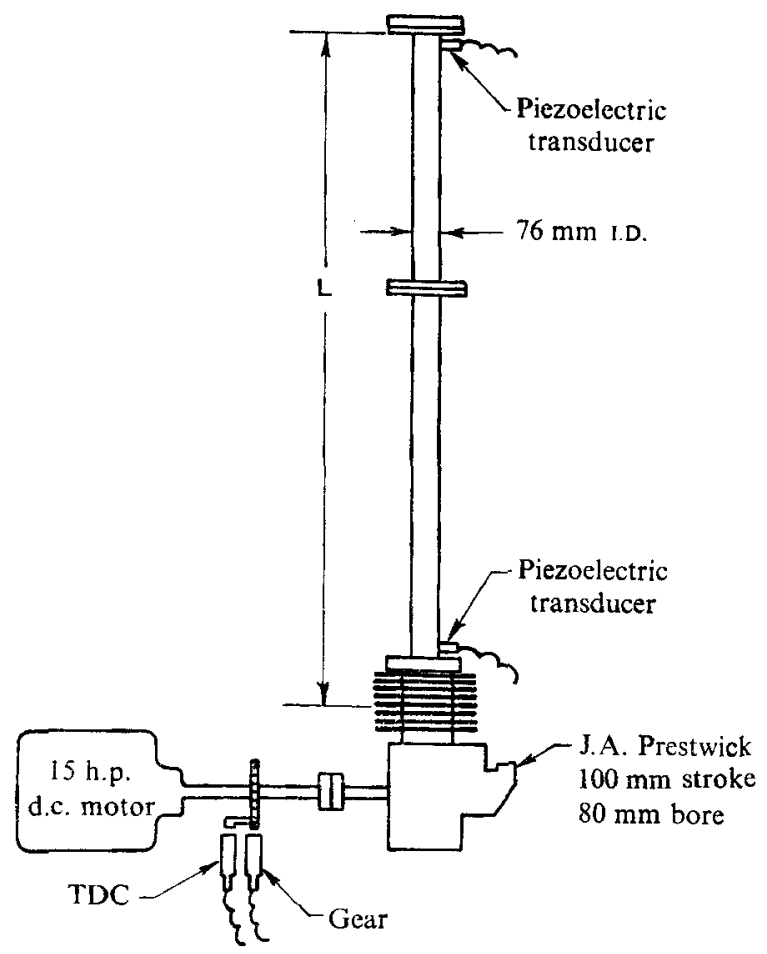

FIGURE 1. Schematic diagram of experimental apparatus. (TDC stands for 'top dead centre'.)

Since the strength of the shock waves produced in this apparatus is in principle limited only by the power available in the drive mechanism, as much power as $15 \mathrm{~h} . \mathrm{p}$. can be dissipated in the resonance tube. Thus, for operation with strong shock waves, the tube must be (and is) water cooled. The wall temperature is measured at $0.75 \mathrm{~m}$ intervals along the tube by thermistors, and the values are recorded automatically with each measurement of the pressure wave form.

The orifice plates used to change the condition at the passive end of the tube are $6.4 \mathrm{~mm}$ thick. The orifices are cut straight through; no attempt is made to bevel the faces of the plates.

The instantaneous pressure is measured at two locations on the side wall of the tube as close to the ends as possible. The transducers are $1.1 \mathrm{~cm}$ diameter quartz piezoelectric gauges $\dagger$ with a nominal resonant frequency of $130 \mathrm{kHz}$.

The data are recorded by a high-speed data acquisition system designed by Prof. D. Coles. Analog data from the pressure transducers are converted to digital data and recorded on magnetic tape alternately with data from a digital crankshaft-angle encoder. Both pressure and shaft-angle data are recorded with a resolution of 1 in 256 . The two channels of pressure data are sampled alternately at a rate of $7500 \mathrm{~s}^{-1}$ per channel.

The data are read, sorted, reduced and plotted using a large batch-processing computer. The records are divided into segments one cycle long, which are

$\dagger$ Kistler Model 606A. 
superposed and averaged. The wave forms from which amplitude data, etc., are obtained are averages of from 2 to 17 cycles and are made up of from 100 to 350 discrete data points.

\section{Procedure for comparing experiment with theory}

For the purpose of comparing experiment with theory it is convenient to transform measured physical variables into the form used in the analysis of $I$, and to compare them directly with the normalized results of $I$. In this section we describe the procedure for processing the experimental data.

In I a normalized frequency $\omega$ is defined in terms of the circular frequency $\varpi$ of the piston motion:

$$
\omega=\varpi L / a_{0},
$$

where $a_{0}$ is the mean sound speed. The value of $a_{0}$ is calculated for each experiment using the mean value of the wall-temperature measurements described in $\S 2$. We treat the length $L$ as unknown, determining it for each experimental configuration (i.e. given piston amplitude and end condition) by adjusting $\omega$ to obtain a best fit between experimental and theoretical response curves introduced in subsequent sections. $\uparrow$ A parameter which measures the difference between $L$ and the actual tube length $L_{0}$, i.e.

$$
\Delta=\left(L-L_{0}\right) / L_{0}
$$

serves to characterize end effects due to two-dimensional flow through the partially open end. Therefore, $\Delta$ is expected to be small for a closed tube and of order $d / L_{0}$, where $d$ is the orifice diameter, for a partially open tube. For small amplitude motions $\Delta$ is positive, the classical value (Rayleigh 1945, vol. 2, p. 183) being approximately $0 \cdot 41 d / L_{0}$.

\subsection{Nonlinear theory}

In $\mathrm{I}$ the one-dimensional motion in a tube aligned with the $x$ axis excited by an oscillating piston at $x=0$ is considered. The dependent variables are expanded in powers of the amplitude $\epsilon$ of the excited motion, where $\epsilon=\delta^{\frac{1}{2}}$ for the closed tube and $\epsilon=\delta^{\frac{1}{3}}$ for the open tube and where $\delta=\omega l / L$ measures the amplitude $l$ of the forcing motion; $\ddagger$ e.g. for the plus and minus Riemann invariants, respectively,

$$
g(\alpha)=\epsilon g_{1}(\alpha)+\ldots, \quad f(\beta)=\epsilon f_{1}(\beta)+\ldots,
$$

where $\alpha$ and $\beta$ are the plus and minus characteristic variables; for the frequency

$$
\omega=\omega_{0}\left(1+\epsilon \omega_{1}+\epsilon^{2} \omega_{2}=\ldots\right),
$$

† For response curves sharply peaked at resonance $L$ can be determined very accurately by this procedure. However, for large damping, when the response curve is flat, the method loses its precision and definitive results are not obtained.

$\ddagger$ For later convenience we also define special values of the expansion parameter at $\omega=\omega_{0}$, the first harmonic resonant frequency, namely, $\delta_{0}=\omega_{0} l / L$ and $\epsilon_{0}=\delta_{0}^{\frac{1}{2}}$ or $\delta_{0}^{\frac{1}{3}}$, respectively. 
where $\omega_{0}=\pi$ for a closed tube and $\omega_{0}=\frac{1}{2} \pi$ for an open tube; for the reflexion coefficient

$$
b=b_{0}\left(1-\epsilon b_{1}-\epsilon^{2} b_{2}+\ldots\right),
$$

where $b_{0}=+1$ for $\omega_{0}=\pi$ and $b_{0}=-1$ for $\omega_{0}=\frac{1}{2} \pi$. The reflexion coefficient is defined by the boundary conditions of the passive end and is the ratio between the reflected wave and the incident wave there: $b=f(\beta) / g(\alpha)$.

In defining the relation for transforming the physical variables to the normalized form used in I, we follow Chester (1964) and modify the definitions of $I$, within the accuracy of the second-order theory, to ensure that far from resonance the nonlinear theory agrees identically with acoustic theory. In particular, we require $(a)$ that the expression for the Riemann invariant $f(t)$ should agree asymptotically with the linear result (e.g. equation (I 2.10) $\dagger$ ) and (b) that, in the limit, the predicted pressure (or equivalently, the sound-speed perturbation $a(t))$ should agree with the acoustic result.

In writing down the relation between $f(t)$ and $f_{1}(t)$ to be used for data reduction, some latitude exists in the precise form of the relation that may be selected because of higher order terms that are neglected in the nonlinear expansion (3). For reasons that will become clear later we choose

$$
f=\epsilon|b|^{\frac{1}{2}} f_{1} \text {. }
$$

Equation (6) agrees with the assumed expansion (3) to the required order. If, in accordance with requirement $(a)$, we specify that far from resonance (in the limit of large $\omega_{1,2}$ and $\left.b_{1,2}\right) f(t)$ should agree with the linear result (I 2.10), then using (6), (T 4.5) and an equivalent result for the open end we obtain

$$
\left.\begin{array}{ll}
\left(4 \pi^{2} \omega_{1}^{2}+b_{1}^{2}\right)^{-\frac{1}{2}}=\epsilon|b|^{\frac{1}{2}}\left[(1-b)^{2}+4 b \sin ^{2}\left(\omega-\omega_{0}\right)\right]^{-\frac{1}{2}} & \text { (closed end) } \\
\left(\pi^{2} \omega_{2}^{2}+b_{2}^{2}\right)^{-\frac{1}{2}}=\epsilon|b|^{\frac{1}{2}}\left[(1+b)^{2}+4 b \sin ^{2}\left(\omega-\omega_{0}\right)\right]^{-\frac{1}{2}} & \text { (open end). }
\end{array}\right\}
$$

Equating separately the terms involving the frequency and reflexion coefficient we obtain the stronger conditions

$$
\left.\begin{array}{lll}
\pi \epsilon \omega_{1}=\sin \left(\omega-\omega_{0}\right), & |b|^{-\frac{1}{2}}(1-b)=\epsilon b_{1} & \text { (closed end), } \\
\frac{1}{2} \pi \epsilon^{2} \omega_{2}=\sin \left(\omega-\omega_{0}\right), & |b|^{-\frac{1}{2}}(1+b)=\epsilon^{2} b_{2} & \text { (open end). }
\end{array}\right\}
$$

These expressions are equivalent to (I 3.10), and agree with it to the required order. Equations (8) are used to calculate $\omega_{1,2}$ from the measured frequency $\omega$ and to calculate the reflexion coefficient $b$ once $b_{1,2}$ have been determined by fitting theory to experiment. It can be seen that the particular expression (6) was chosen in order to ensure that in (8) the equations for $\omega_{1,2}$ would be independent of $b$, and vice versa.

In order to compare the measured pressures with the solutions $f_{1}(t)$ of 1 , we first calculate the normalized sound speed $a(t)$ from $p(t)$ assuming isentropicity (but not linearity):

$$
a(t)=(1+p(t))^{(\gamma-1) / 2 \gamma}-1
$$

$\dagger$ The notation (I 2.10) is used to refer to equation (2.10) in the first part of this series (Jimenez 1973). 
Next, a relation between $a(t)$ and $f_{1}(t)$ is needed. As in the preceding paragraph, a certain amount of discretion may be exercised here because of the higher order terms neglected in the nonlinear theory, and in this case the choice is made to ensure that requirement $(b)$ above is satisfied. Furthermore, for the purposes of the comparison it is convenient to consider only the pressure at the piston when dealing with the open tube, and the pressure at the passive end when dealing with the closed tube. Therefore (cf. (16) and (17))

$$
a(t)=\left\{\begin{array}{ll}
\frac{1}{2}(\gamma-1) \epsilon|b|^{-\frac{1}{2}}(1+b) f_{1}(t) & \text { (closed end), } \\
\frac{1}{2}(\gamma-1) \epsilon|b|^{-\frac{1}{2}}\left[(1-b)^{2}-4 b \sin ^{2}\left(\omega-\omega_{0}\right)\right]^{\frac{1}{2}} f_{1}(t) & \text { (open end), }
\end{array}\right\}
$$

where $\omega$ and $b$ are given by (8). Equations (10) agree with the equivalent relations (I 3.2) and (I 3.9) to second and third order, respectively.

Equations (9) and (10) are used to calculate $f_{1}$ from the measured pressure. Since they are applied before $b_{1,2}$ are determined by comparison of experiment with theory, the data reduction procedure is necessarily iterative.

\subsection{Nonlinear theory: special considerations for the open end}

It became clear very early in the analysis of the data that the amplitude of the wave form in the open tube, plotted against frequency, did not follow the theoretical curves for $b_{2}=$ constant, but instead seemed to suggest that $b_{2}$ increased with amplitude $\left(f_{1 \text { max }}\right)$. Further investigation confirmed that $b_{2}$ was in fact proportional to $f_{1 \max }$. This result agrees precisely with the conclusion of Ingard \& Ising (1967) that for large amplitude motions the acoustic impedance of an orifice is purely resistive and is proportional to the amplitude of the flow velocity through the orifice. Indeed, they found that the pressure amplitude $\tilde{p}_{\max }$ and velocity amplitude $\tilde{u}_{\max }$ at the orifice were related by a unique value of a 'wave pressure coefficient', say $C_{p}$,

$$
C_{p} \equiv \tilde{p}_{\max } /\left(\frac{1}{2} \rho_{0} \tilde{u}_{\max }^{2}\right) \doteq \mathbf{2} \cdot \mathbf{0} .
$$

Now, for an open tube the orifice velocity is equal to the acoustic disturbance velocity $\tilde{u}=a_{0} u$ at the passive end, and

$$
u=f(\beta)(1-b) / b \text {. }
$$

The specific acoustic resistance $r=\tilde{p} / \rho_{0} a_{0} \tilde{u}$ is given by

$$
r=(1+b) /(1-b)
$$

so (11) becomes (ef. equation (6))

$$
2 b(1+b) /(1-b)^{2}=C_{p} f_{\max }=\epsilon|b|^{\frac{1}{2}} C_{p} f_{1 \max }
$$

Combining (14) and (8) and retaining only the largest terms gives

$$
\epsilon b_{2}=2 C_{p} f_{1 \max }
$$

which conforms to our preliminary observations. Therefore, in presenting the theoretical response curves (amplitude $v s$. frequency), we plot curves of constant $C_{p}$ rather than of constant $b_{2}$ as in I. $C_{p}$ is treated here as an adjustable parameter to be determined by our experiments. 


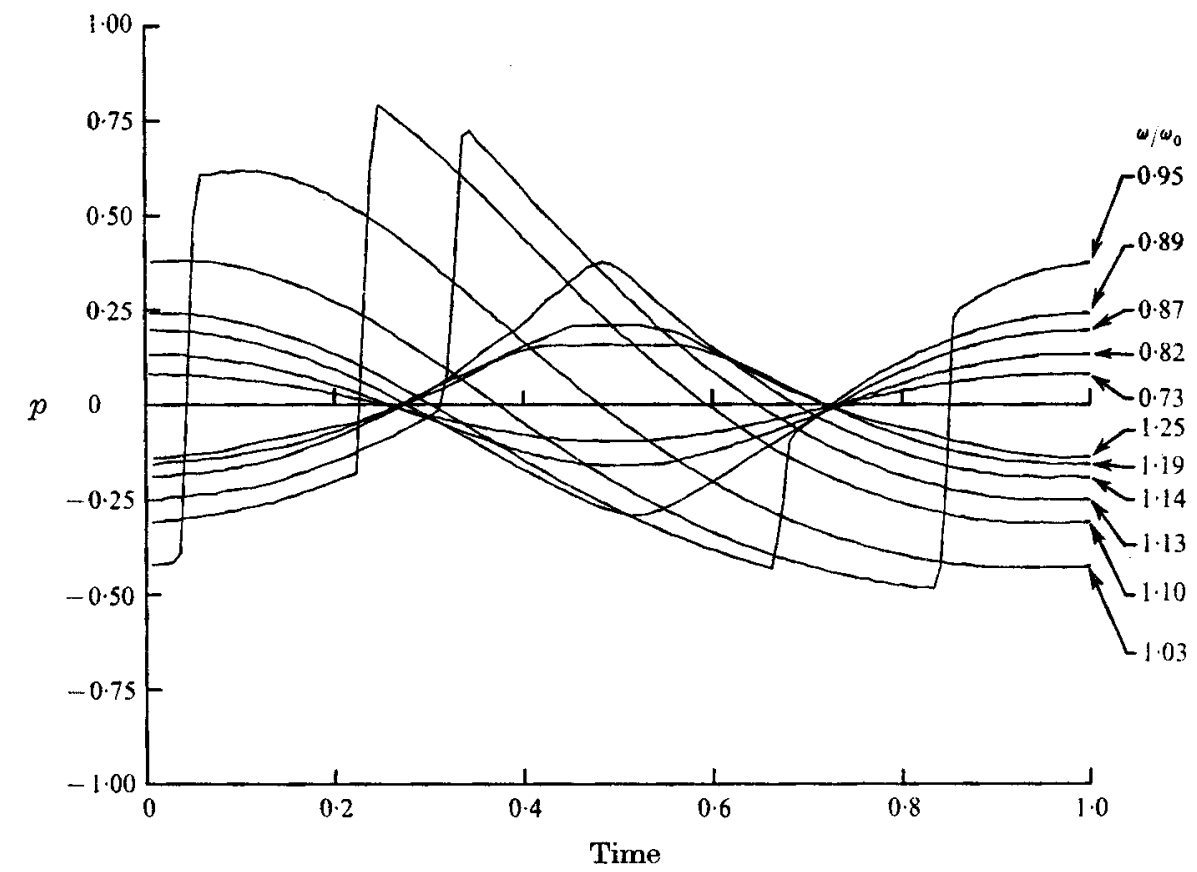

FrgURE 2. Normalized pressure wave form at closed end for $\omega_{0}=\pi$. Time measured from maximum amplitude of piston (TDC). File $283 \cdot 05$ (cf. table 3 ).

\subsection{Linear theory}

For orifice area ratios $A_{0} / A$ greater than about $\frac{1}{4}$ but smaller than about $\frac{2}{3}$ the amplitude of the wave motion in our experiments is sufficiently small that there is little difference between the amplitudes predicted by the linear and nonlinear theories. Therefore, for such cases we simply evaluate the reflexion coefficient $b$ by comparing the measured amplitude $a_{\text {max }}$, calculated from (9), with the prediction of acoustics; for the closed end (for which pressure is measured at the passive end)

$$
a_{\max }=\frac{1}{2}(\gamma-1) \epsilon^{2}(1+b)\left[(1-b)^{2}+4 b \sin ^{2} \omega\right]^{-\frac{1}{2}}
$$

and for the open end (pressure measured at the piston)

$$
a_{\max }=\frac{1}{2}(\gamma-1)\left[(1-b)^{2}+4 b \cos ^{2} \omega\right]^{\frac{1}{2}}\left[\left(1-b^{2}\right)+4 b \sin ^{2} \omega\right]^{-\frac{1}{2}} .
$$

\section{Results for closed tubes}

\subsection{Solid end}

In figure 2 are examples of the now-familiar (e.g. Chester 1964) wave forms observed for nonlinear oscillations near resonance in closed tubes $\left(\omega_{0}=\pi\right)$. Below a lower critical frequency and above an upper critical frequency the wave forms are smooth. As the frequency approaches the resonant frequency the amplitude increases but the phase difference between the pressure response and the piston motion is virtually constant. On the other hand, at frequencies intermediate between the two critical frequencies, the wave forms are discontinuous 


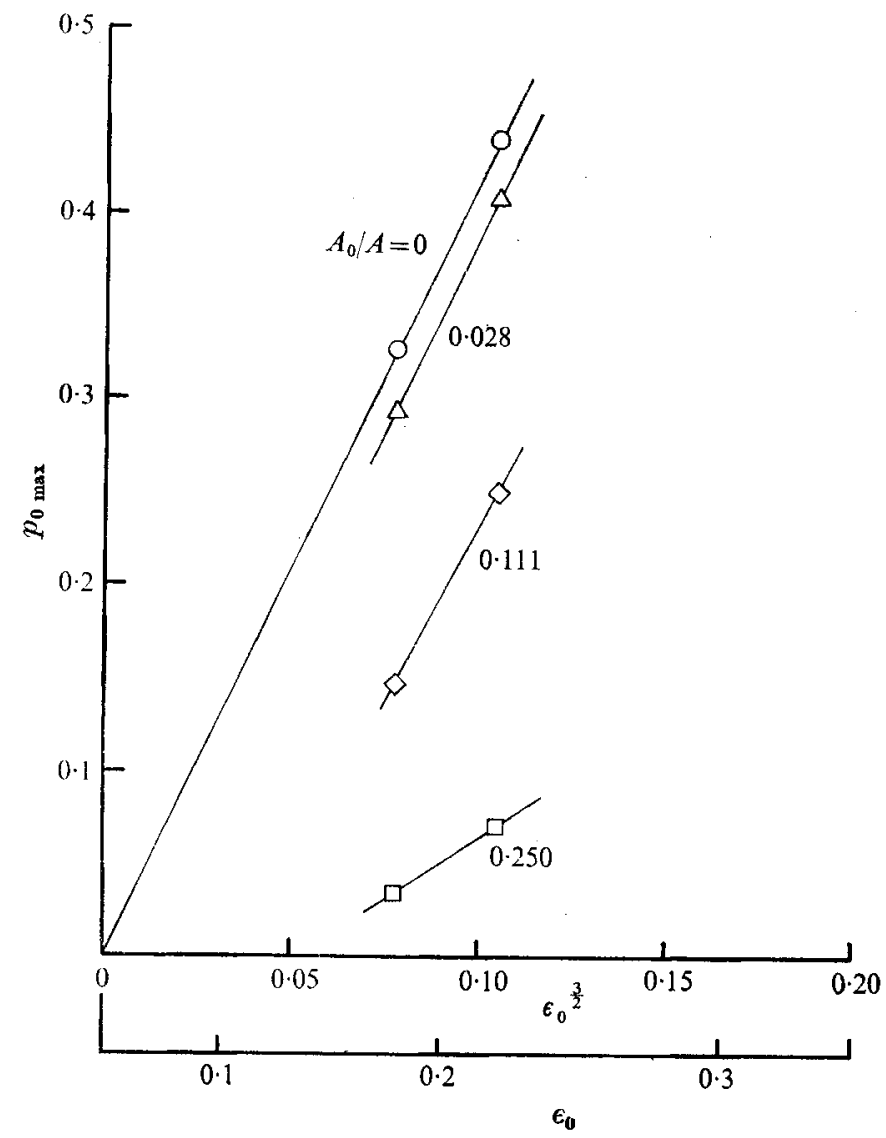

Figure 3. Normalized pressure amplitude at resonant frequency versus $\epsilon_{\text {w }}$ in closed tube $\left(\omega_{0}=\pi\right)$.

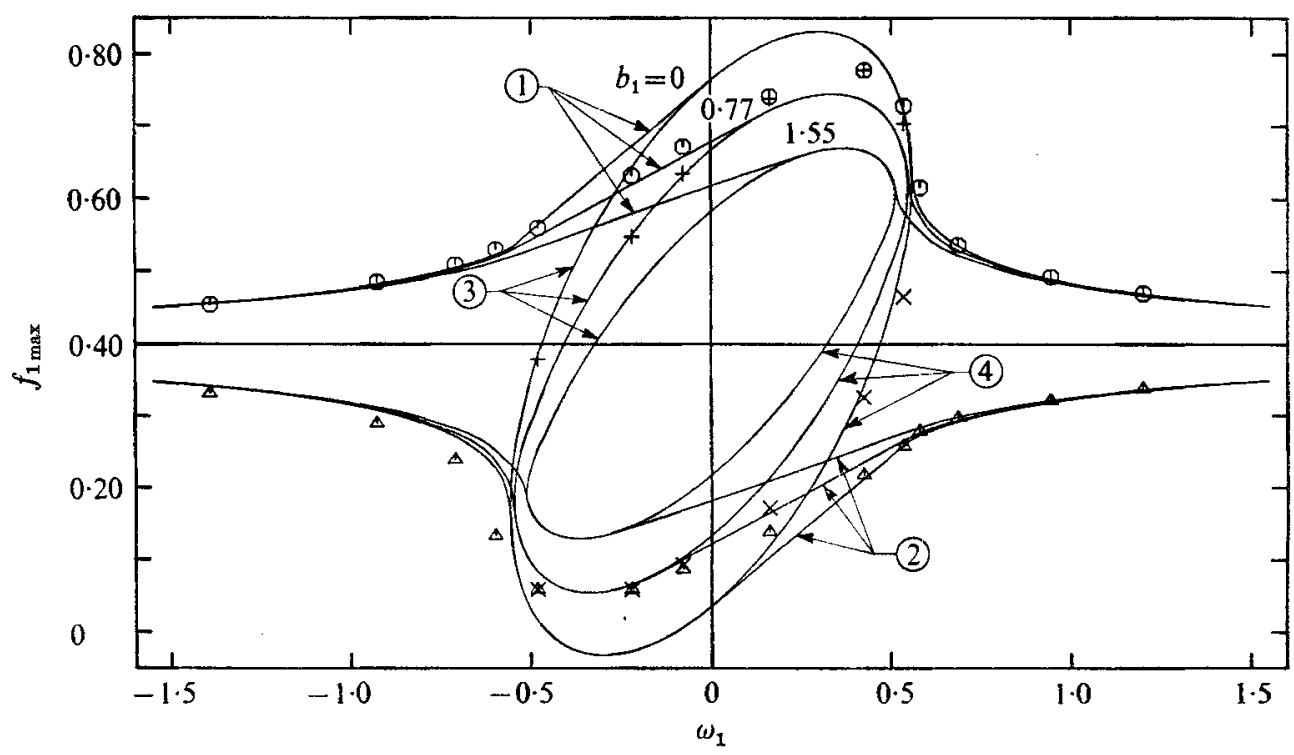

FigURe 4. Response curve for closed tube. - - nonlinear theory: (1), maximum value of $f_{1}$; (2), minimum value of $f_{1} ;$ (3), $f_{1}$ just behind discontinuity ; (4), $f_{1}$ just ahead of discontinuity. Experiment: $O$, maximum $f_{1} ; \Delta, \operatorname{minimum} f_{1} ;+, f_{1}$ behind shock; $\times, f_{1}$ ahead of shock. File $94 \cdot 19$ (cf. table 2). 

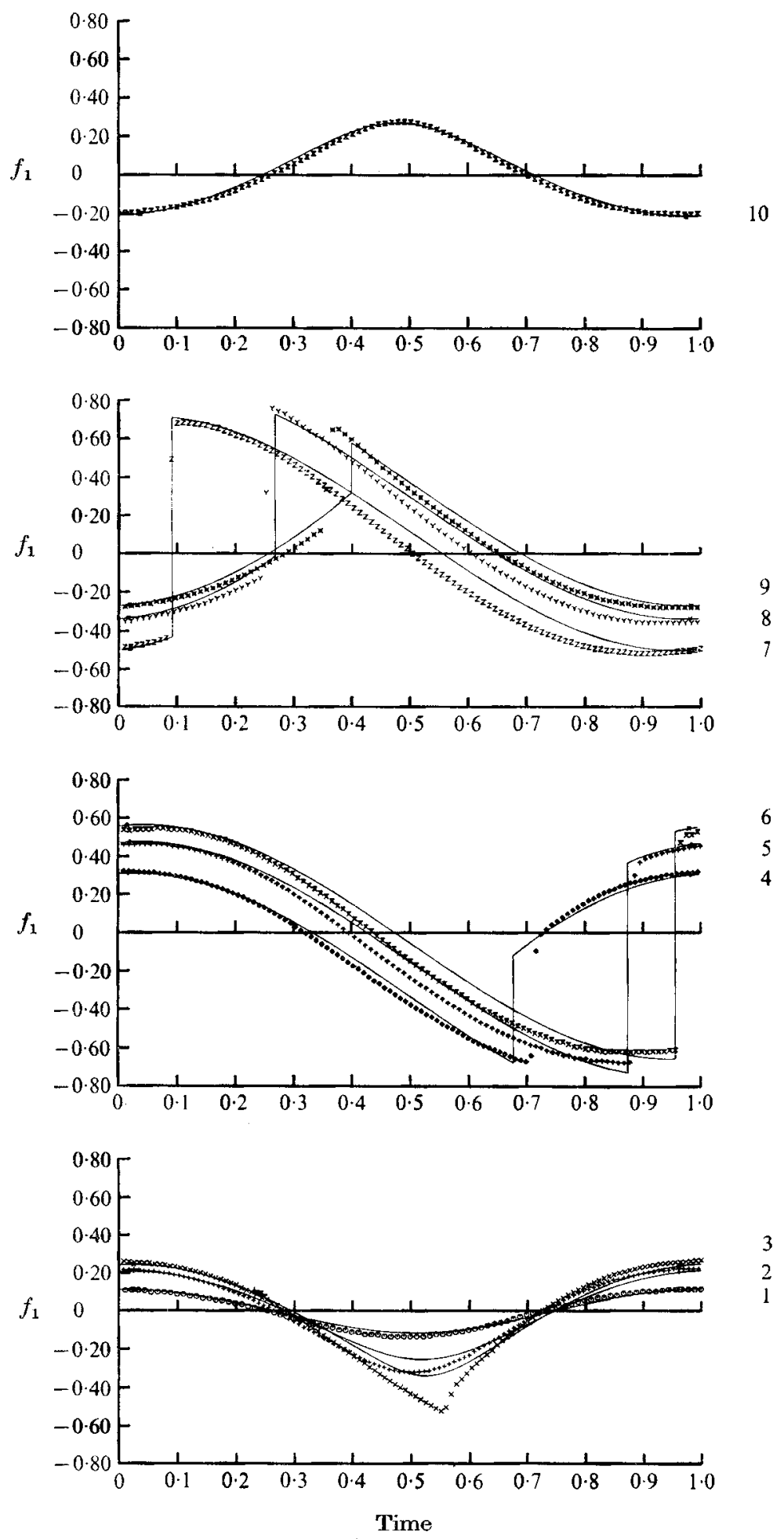

Figure 5. Comparison of the observed (symbols) and predicted (solid line) wave forms for the experiment of figure $4 . b_{1}=0.50$. Curves: $1, \omega_{1}=-1.39 ; 2, \omega_{1}=-0.71 ; 3, \omega_{1}=-0.60$; $4, \omega_{1}=-0.48 ; 5, \omega_{1}=-0.22 ; 6, \omega_{1}=-0.08 ; 7, \omega_{1}=0.16 ; 8, \omega_{1}=0.43 ; 9, \omega_{1}=0.54 ;$ 10, $\omega_{1}=0 \cdot 69$. File $94 \cdot 19$. 


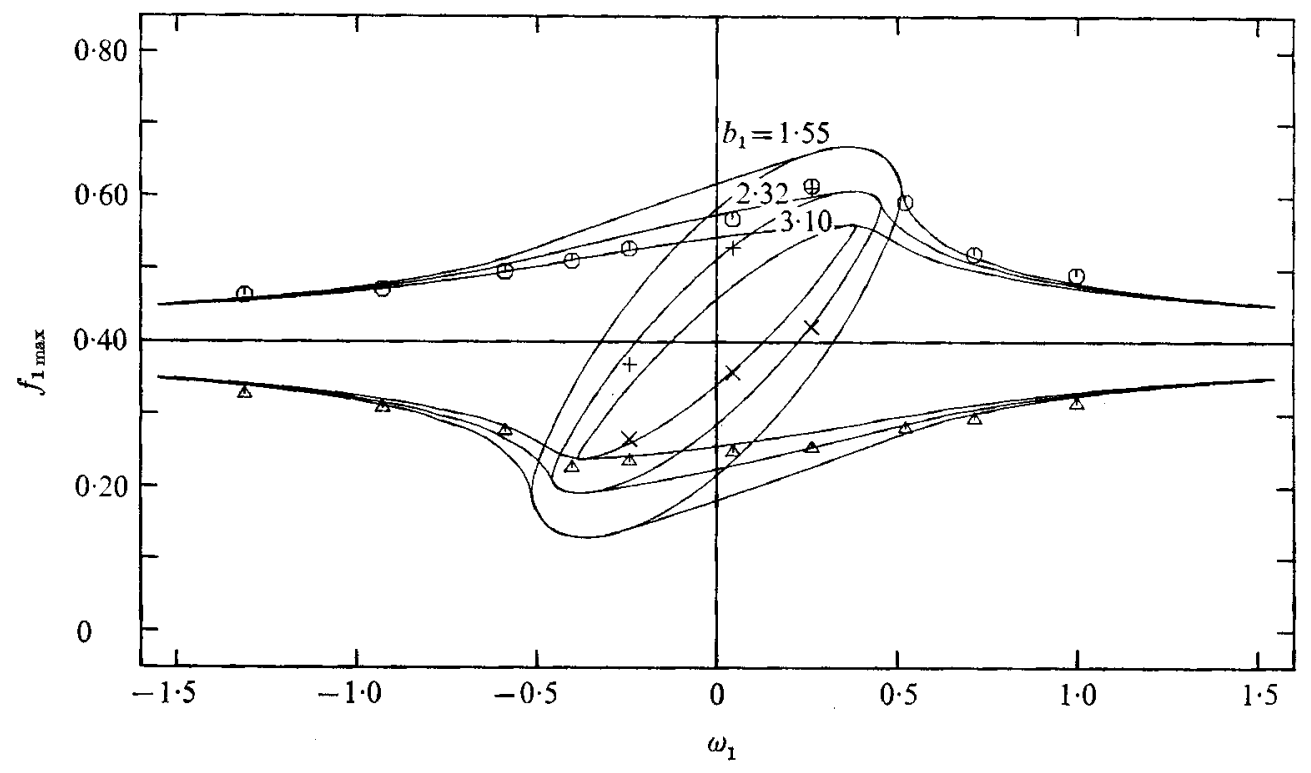

Figure 6. Response curve for partially closed end for $\omega_{0}=\pi$. Orifice diameter $=2.5 \mathrm{~cm}$. Cf. figure 4 for legend. File 283.07.

(shock waves occur) and the phase angle changes continuously by $180^{\circ}$ as the frequency band is traversed.

The effect of the amplitude of the driving motion has been investigated by conducting experiments with two different tube lengths. The dependence of the pressure amplitude at resonance $\left(\omega=\omega_{0}\right)$ on $\epsilon_{0}$ is indicated in figure 3 .

Figure 4 is a comparison of theoretical and experimental response curves for a closed tube; there are four characteristic pressures on the wave forms of figure 2 which are of interest, namely, the absolute maximum and minimum pressures and pressures just behind and just ahead of the shock wave, when it occurs. These four pressures are separately plotted to form the characteristically eggshaped response curves of figure 4. Comparison between experiment and theory on that figure is judged to imply a best-fit value $b_{1}=0.50$. The quantitative disagreement apparent especially near the critical frequencies is typical of all experiments conducted with our apparatus; measured critical frequencies differ from those predicted by $\frac{1}{2}-1 \%$ and consistently occur further from the resonant frequency than is predicted by theory. Therefore, in view of the very sensitive dependence of amplitude on frequency near the critical frequencies, the observed amplitudes in that region are always substantially larger than those predicted.

After choice of an appropriate value of $b_{1}$, a more detailed comparison between theory and experiment may be made by comparing the wave forms. In figure 5 the theoretical wave form is plotted as a solid line while each plotted symbol represents the mean of 7-11 experimental data points, depending on the frequency, taken one each cycle (cf. §2). It is seen that, to a large extent, the theory contains the qualitative features exhibited by the experiments. Furthermore, it is our experience that changes of \pm 0.05 in $b_{1}$ cause easily observable deterioration of the 

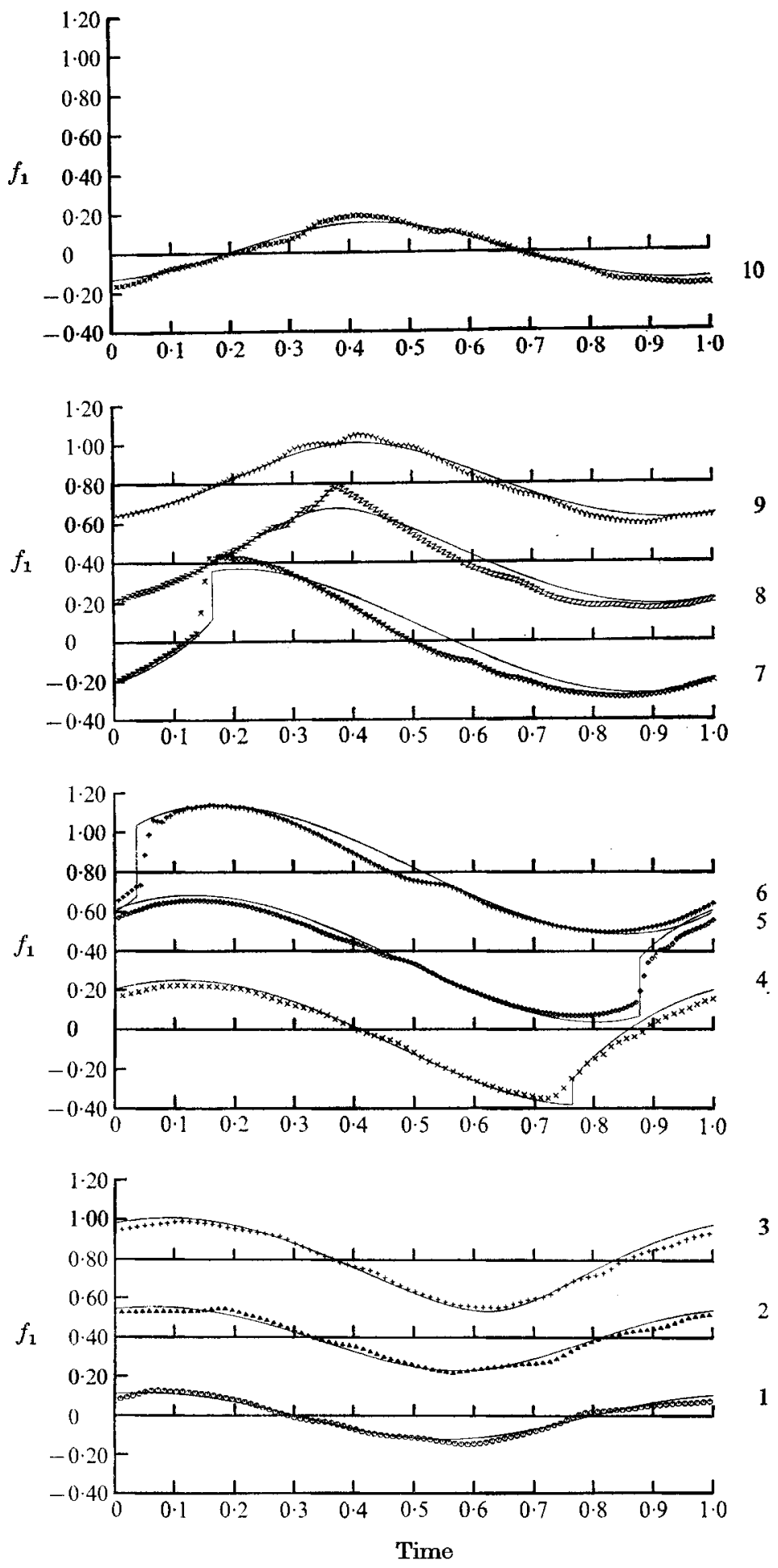

FrGURE 7. Comparison of the observed (symbols) and predicted (solid line) wave forms for the experiment of figure $6 . b_{1}=2 \cdot 6$. Curves: $1, \omega_{1}=-1.30 ; 2, \omega_{1}=-0.91 ; 3, \omega_{1}=-0.57$; $4, \omega_{1}=-0.38 ; 5, \omega_{1}=-0.22 ; 6, \omega_{1}=0.07 ; 7, \omega_{1}=0.29 ; 8, \omega_{1}=0.55 ; 9, \omega_{1}=0.74 ;$ 10, $\omega_{1}=1 \cdot 02$. File 283.07. 


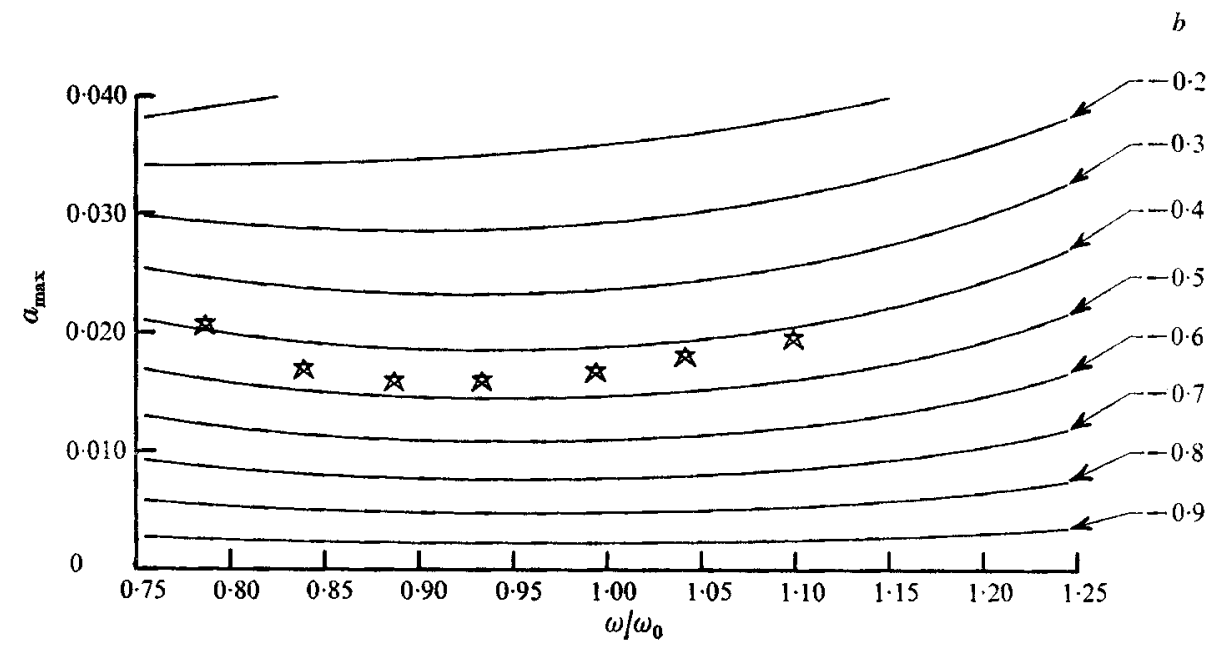

Figure 8. Comparison of experiment with linear theory for $\omega_{0}=\pi$. Orifice diameter $=5.1 \mathrm{~cm}$. — , theory; , experiment. File 283.10.

agreement exhibited in figure 5. Therefore, in view of (8), it is concluded that the reflexion coefficient $b$ is determined with a resolution of approximately $\pm 0 \cdot 01$.

\subsection{Orifice plates at end}

Even a small departure from a solid wall at the end of the tube has a substantial effect on the amplitude of the resulting motion. Figure 6 is the response curve for a tube terminated with a $2.5 \mathrm{~cm}$ diameter orifice (orifice area ratio $A_{0} / A=0 \cdot 11$ ) in an experiment which is otherwise identical with that of figure 4 . The bestfit value of $b_{1}$ has increased to $2 \cdot 6$, implying (cf. equation (8)) a reflexion coefficient at resonance of only $b_{0} \equiv b\left(\omega_{0}\right)=0.57$. It is seen from the measured wave forms (figure 7) that not only does the presence of an orifice decrease the amplitude of the shock waves but it also seems to thicken them. It is possible that the shocks are dispersed by refraction or scattering in the neighbourhood of the orifice plate, and this interesting effect should be investigated further.

Figure 8 shows the result of introducing a still larger orifice $(5 \cdot 1 \mathrm{~cm}$ diameter; $\left.A_{0} / A=0.44\right)$; the amplitude is so low that comparison with linear theory suffices.

The values of the parameters determined from our experiments with $\omega_{0}=\pi$ are summarized in table 2 and in figures 3, 9 and 10. Figure 9 shows the variation of the transmission parameter $b_{1}$ of the nonlinear theory with area ratio $A_{0} / A$. Figure 10 shows the reflexion coefficient $b_{0}$ at the resonant frequency determined by both nonlinear and linear theory for all the experiments conducted with $\omega_{0}=\pi$. It is notable that, in qualitative agreement with the nonlinear behaviour in open tubes already alluded to and described more fully below, the reflexion coefficient of each of the orifice plates tested is larger for larger amplitude motion than for smaller amplitude motion. Furthermore, the condition for zero reflexion and therefore for purely progressive waves in the tube occurs with a surprisingly small orifice $\left(A_{0} / A \div 0 \cdot 25\right)$. 


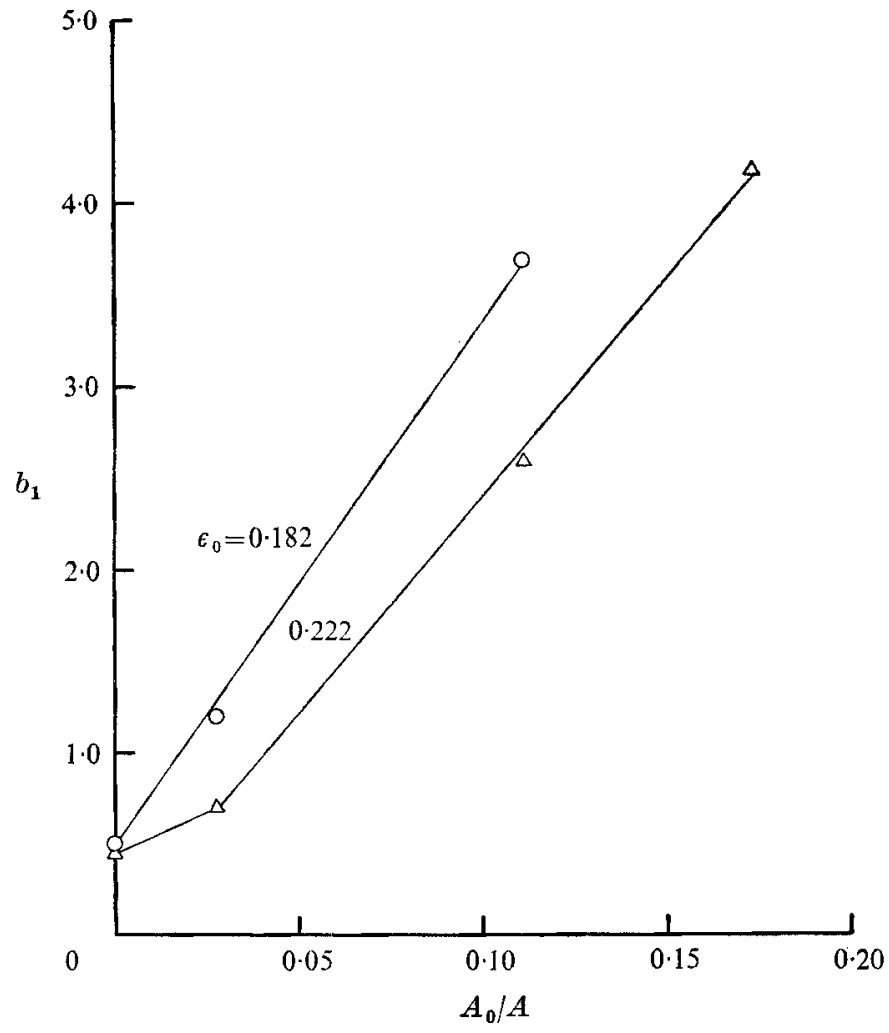

FIguRe 9. Transmission parameter of nonlinear theory verus orifice area ratio for $\omega_{0}=\pi$.

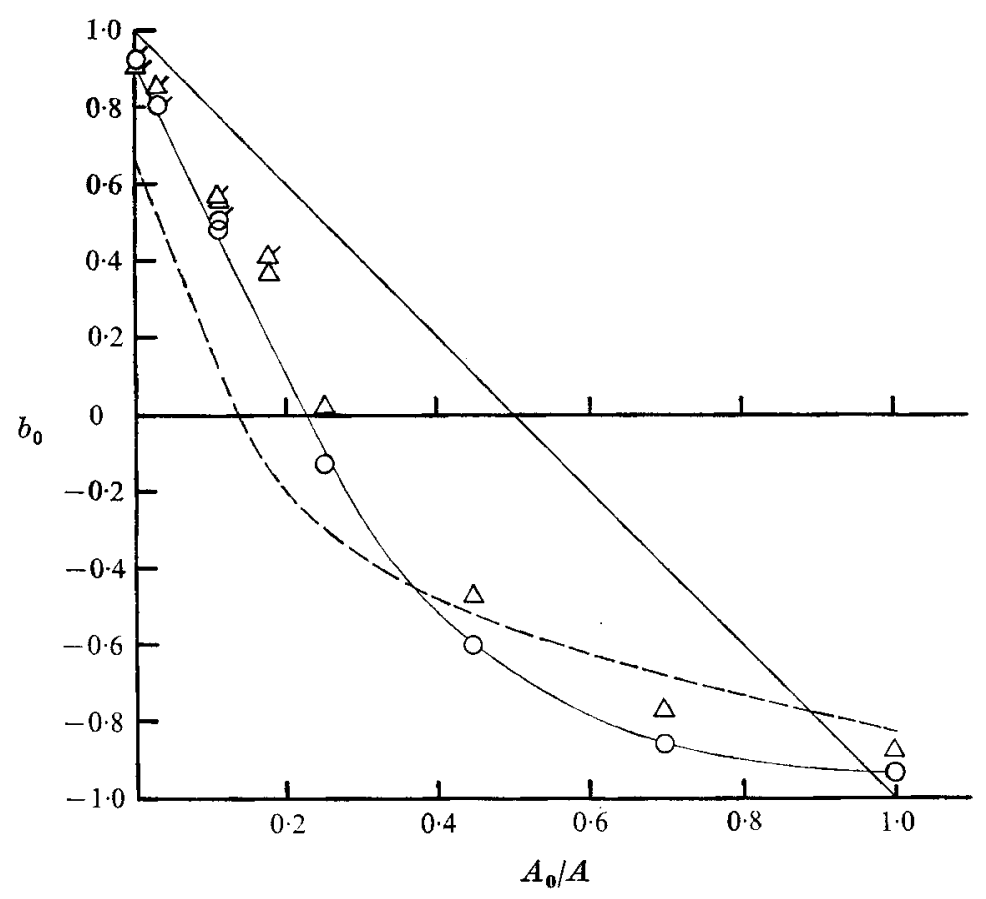

FIGURe 10. Reflexion coefficient at resonant frequency versus orifice area ratio for $\omega_{0}=\pi$. $\triangle, L=3.2 \mathrm{~m}, \epsilon_{0}=0.22 ; \mathrm{O}, L=4.7 \mathrm{~m}, \epsilon_{0}=0.18$. Flags: $\sigma$, data from nonlinear theory; $O$, data from linear theory. - . , open-end results. 


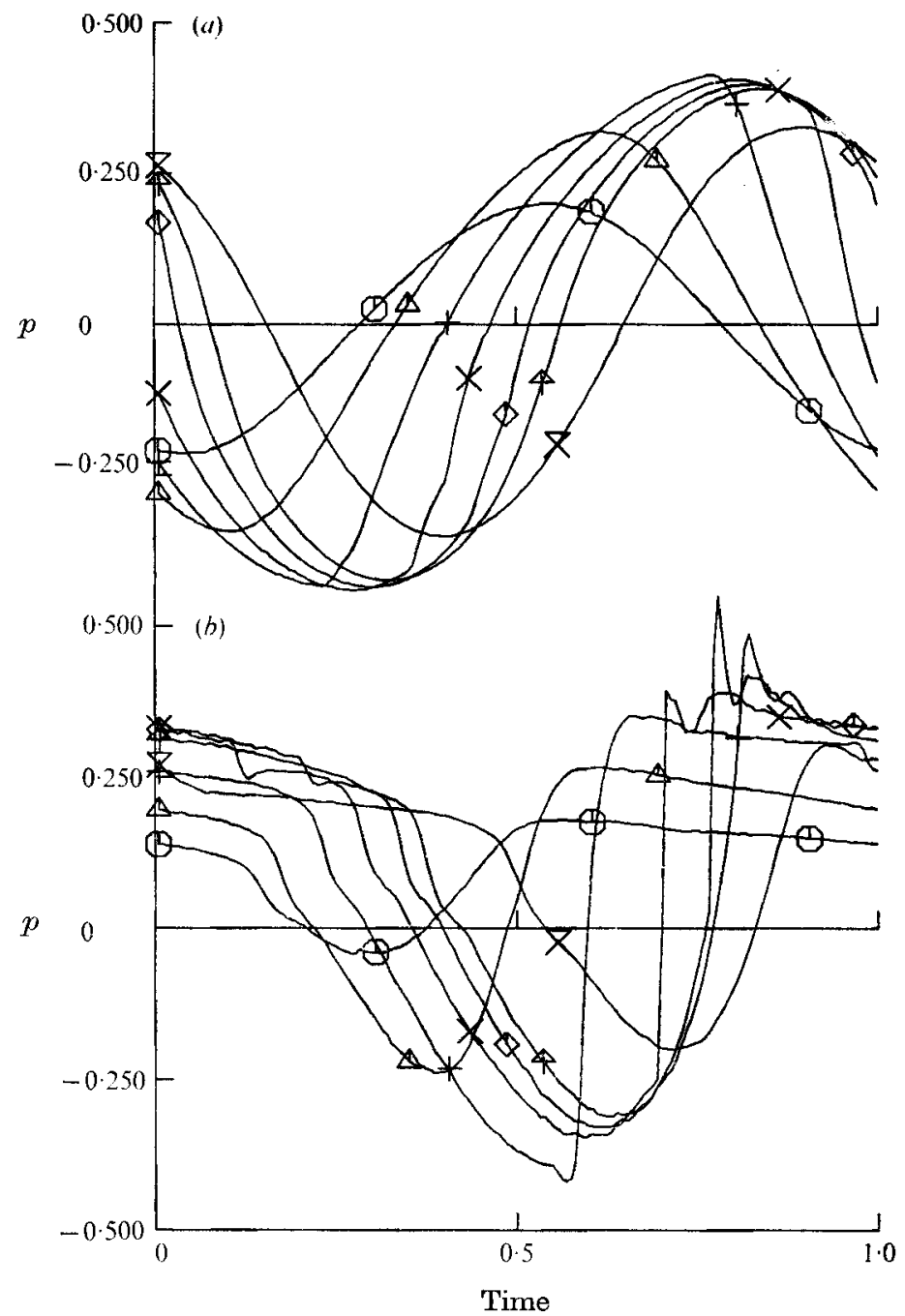

FIGURE 11. Normalized pressure wave form in open tube $\left(\omega_{0}=\frac{1}{2} \pi\right) .(a)$ Pressure at piston end. (b) Pressure at open end. Time measured from maximum amplitude of piston (TDC). $\omega / \omega_{0}$ : $0.0 .84 ; \Delta, 0.90 ;+, 0.95 ; \times, 0.98 ; \diamond, 1.01 ; \uparrow, 1.03 ; \not, 1.09$. File $94 \cdot 03$ (cf. table 3 ).

\section{Results for open tubes}

\subsection{Open end}

Typical pressure wave forms observed at both the piston end and the passive end of an open tube $\left(\omega_{0}=\frac{1}{2} \pi\right)$ are shown in figure 11. Most notable is the fact that the pressure at the open end is by no means zero, as would be the case if the reflexion coefficient were $b=-1 \cdot 0\left(b_{2}=0\right)$; in fact, the pressure amplitude at the open end is of the same magnitude as that at the piston, and for the experiment exhibited in figure 11, the wave forms there are discontinuous. That is, even though the wave at the piston is smooth and continuous, its compressive portions steepen to form shocks in less than one tube length. Therefore, repeated shock 


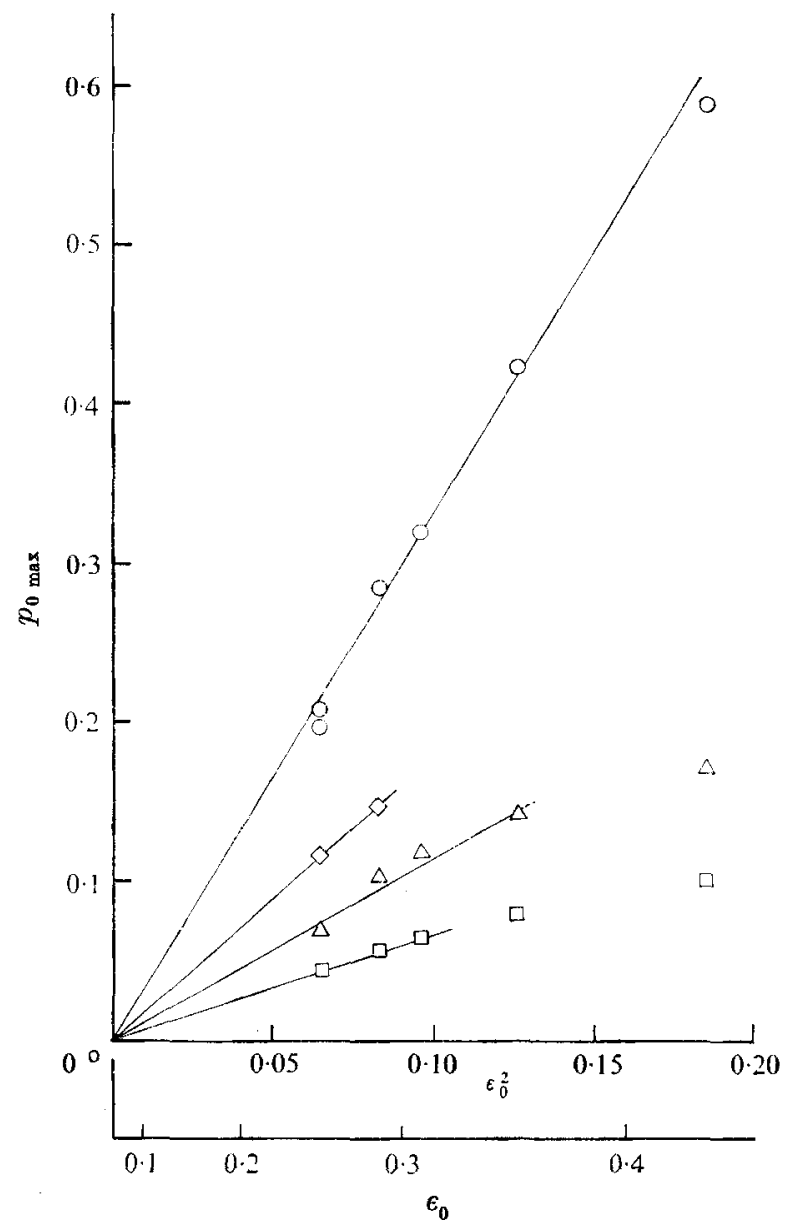

FrgURE 12. Normalized pressure amplitude at resonant frequency versus $\epsilon_{0}$ in open tube $\left(\omega_{0}=\frac{1}{2} \pi\right) . A_{0} / A: \bigcirc, 1.00 ; \diamond, 69 ; \triangle, 0 \cdot 44 ; \square, 0 \cdot 25$.

waves are emitted from the open end of the tube, and the machine is a remarkably effective noisemaker ! Despite the energy loss by radiation which inevitably accompanies such behaviour, the amplitude of the motion is extremely large. The wave form at the piston exhibits a characteristic asymmetry which is untypical of the behaviour of any second-order theory, but which, as we shall see, is predicted by the third-order theory of $I$. These last two facts constitute strong evidence that finite amplitude motion in an open tube is a third-order phenomenon.

The mean half-amplitude of the pressure on the piston at resonance $\left(\omega=\omega_{0}\right)$ is plotted versus the amplitude of the exciting motion in figure 12. It is seen that the pressure varies very nearly as $\epsilon^{2}$. This in turn implies a very specific, though not necessarily simple, dependence of the reflexion coefficient on amplitude. The reflexion coefficient, or more properly the 'wave pressure coefficient' defined by (11), is determined by transforming the piston pressure to $f_{1}$ as described in $\S 3$, plotting the amplitude versus the normalized frequency and comparing with 


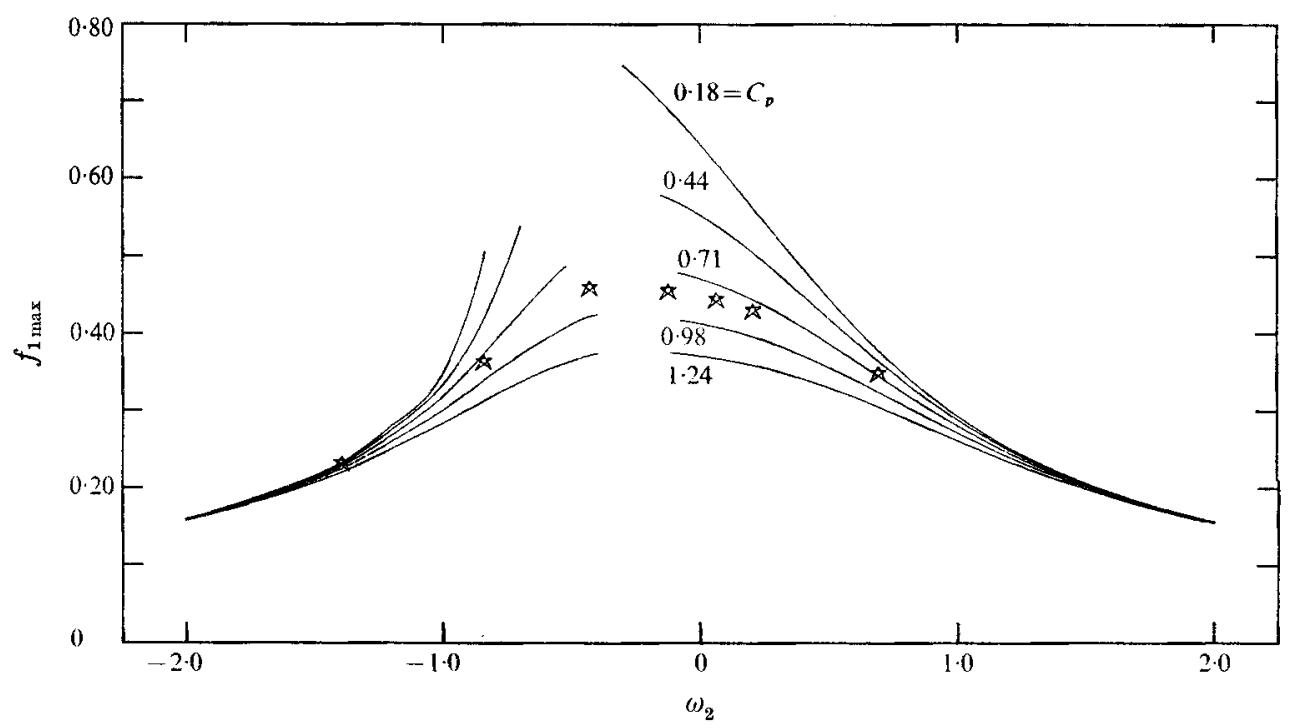

Figure 13. Response curve for experiment of figure 11; open tube. —_, theory. *, experiment. File $94 \cdot 03$.

the theoretical response curves for $C_{p}=$ constant. An example of this is shown in figure 13. After determining the best-fit value of $C_{p}(0 \cdot 76$ for the case shown) the experimental and theoretical wave forms may be compared (figure 14). As noted above, the qualitative features of the observed wave forms are exhibited by the theory. The largest distortions of the piston pressure wave form occur, of course, when shocks form somewhere in the tube (as in figure 11). Unfortunately, in most of these cases the theory is inapplicable so solution of the equations of motion is not carried through (cf. $\S 5$ of $\mathrm{I}$ ). Therefore, comparison between experiment and theory can not be made in many of the most interesting cases. The quantitative disagreement between the observed and predicted wave forms which does occur in figure 14, particularly in the phase shift between experiment and theory, is typical of all the comparisons that have been made. Why the predicted wave form consistently lags the observed one is not understood.

Figure 15 exhibits the dependence of the wave pressure coefficient at the open end on $\epsilon_{0}$. It will be recalled (cf. $\S 3$ ) that for fixed $\epsilon_{0} C_{p}$ remains constant as the wave amplitude $f_{1 \text { max }}$ varies in the neighbourhood of a resonance peak (figure 13). However, figure 15 shows that $C_{p}$ is not independent of the forcing amplitude $\epsilon_{0}$.

In order to compare the results with those of Ingard \& Ising (1967) obtained under very different conditions, orifice Reynolds numbers $R e$ based on the maximum velocity amplitude in the orifice have been formed for the two experiments, and $C_{p}$ is also plotted versus $R e$ in figure 15 . For the present experiments the perturbation velocity amplitude at the open end is calculated from the measured amplitude using (12) and then the orifice Reynolds number is calculated:

$$
R e=\left(a_{0} d / \nu\right) u_{\max }
$$



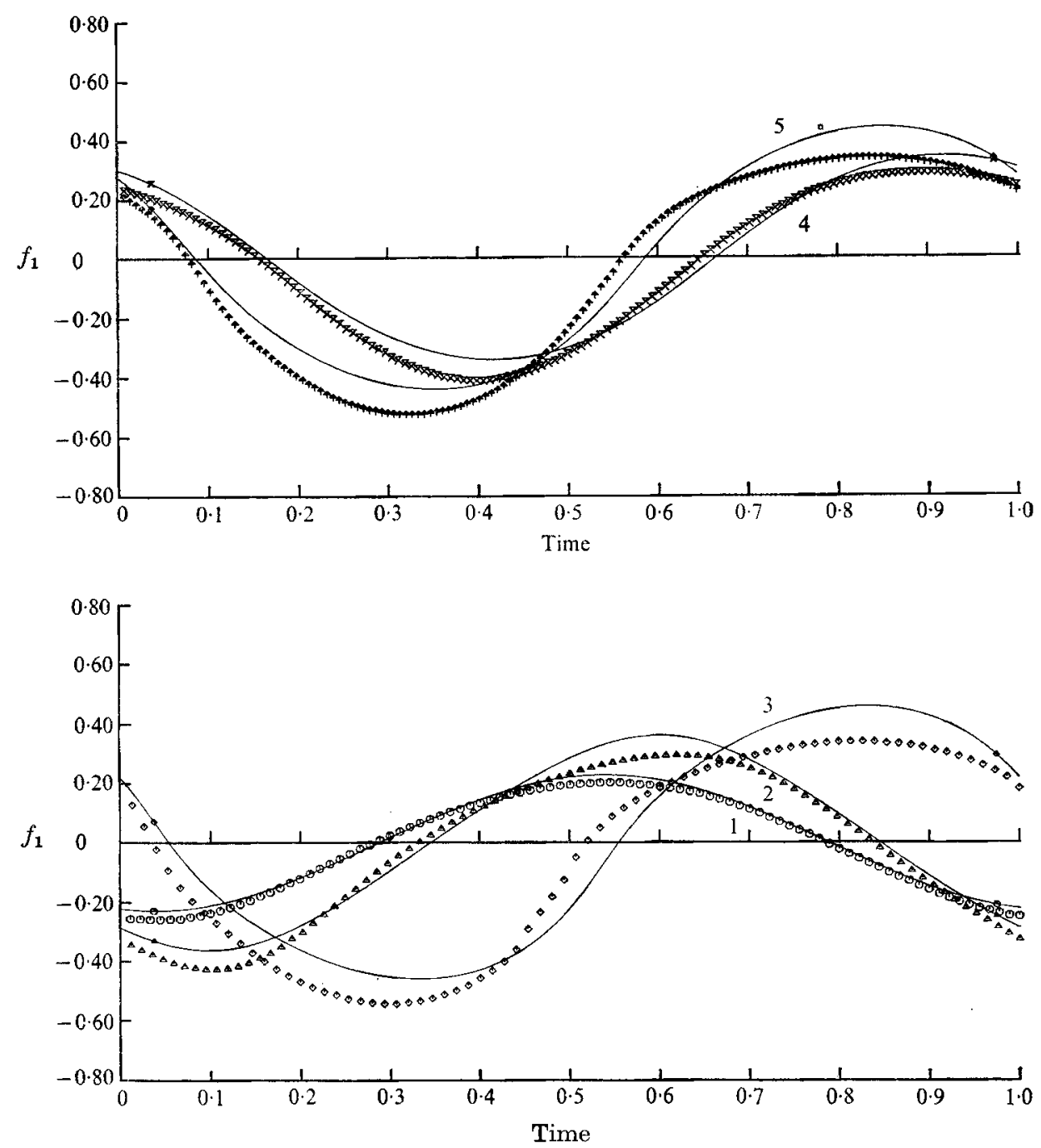

FIGURE 14. Comparison of the observed (symbols) and predicted (solid line) wave forms for the experiment of figure 13. $C_{p}=0.76$. Curves: $1, \omega_{2}=-1.40 ; 2, \omega_{2}=-0.84 ; 3, \omega_{2}=0.06$; $4, \omega_{2}=0 \cdot 69 ; 5, \omega_{2}=0 \cdot 20$. File $94 \cdot 03$.

where $v$ is the kinematic viscosity of air. Ingard \& Ising measured the orifice velocity so $R e$ is calculated directly from their tabulated results. It should be emphasized that $R e$ is used here primarily as a measure of amplitude. Because there are so many other differences between the two experiments (e.g. frequency, method of excitation, etc.), we do not mean to imply that the differences between the two experiments apparent in figure 15 are due solely to viscous effects. However, the present results do indicate that Reynolds number effects should be examined more carefully, both with the present apparatus and with others. 


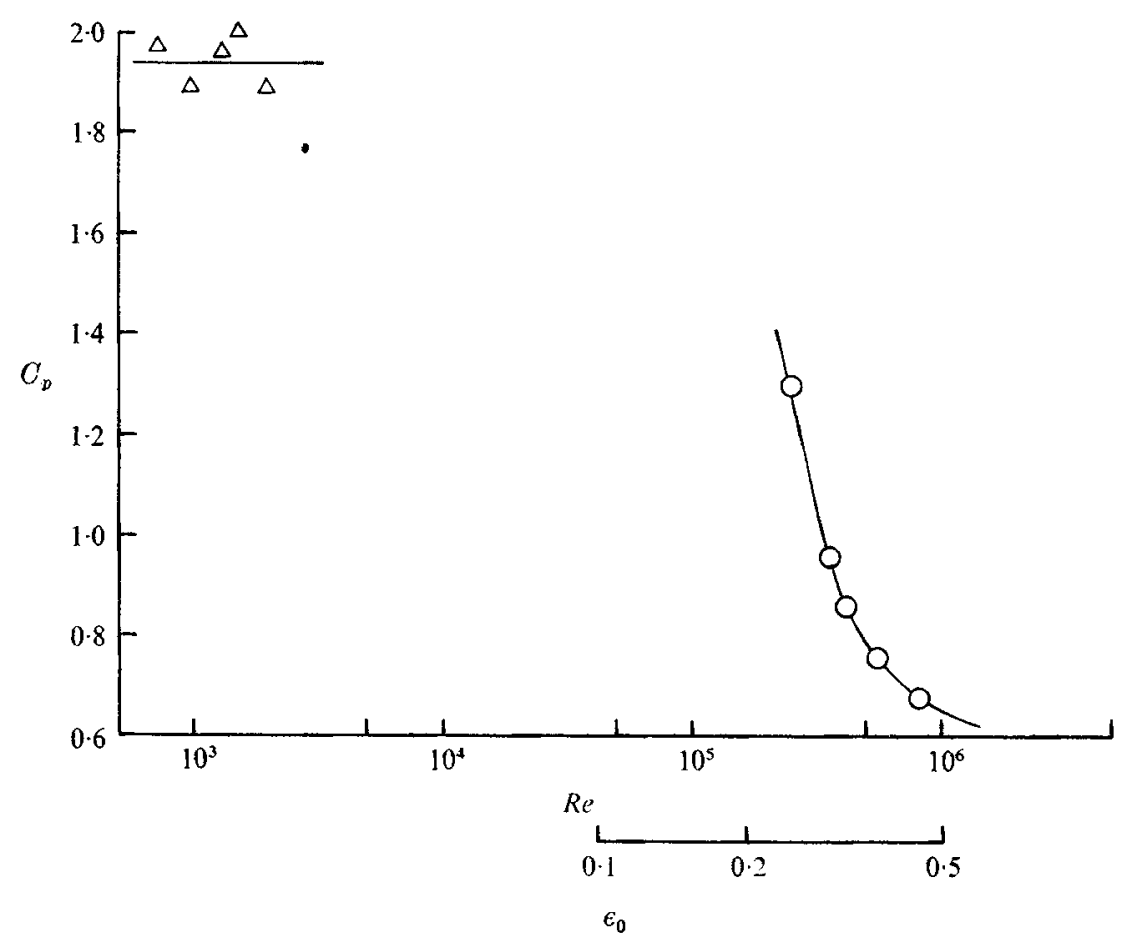

Figure 15. Wave pressure coefficient for an open tube versus amplitude $\epsilon_{0}$ and orifice Reynolds number Re. O, present data; $\triangle$, data from Ingard \& Ising (1967).

\subsection{Orifice plates at end.}

As shown in figure 16, which is plotted on the same scale as figure 11, the addition of solidity to the end boundary condition is effective in reducing the amplitude of oscillations at $\omega_{0}=\frac{1}{2} \pi$; the experiment of figure 16 is identical to that of figure 11 except that a plate with a $5 \cdot 1 \mathrm{~cm}$ diameter orifice $\left(A_{0} / A=0 \cdot 44\right)$ caps the passive end. Even though the wave forms are highly distorted, comparison of the wave amplitude at the piston with the predictions of linear theory is sufficient to determine the reflexion coefficient of the orifices; figure 17 is a typical response curve used for this purpose.

The results of our study of the open tube $\left(\omega_{0}=\frac{1}{2} \pi\right)$ with and without orifice plates are summarized in table 3 and in figures 12 and 18. The reflexion coefficient at the resonant frequency, determined for the complete set of experiments with $\omega_{0}=\frac{1}{2} \pi$, is shown in figure 18. As with the studies at $\omega_{0}=\pi$ (figure 10) the reflexion coefficient increases with increasing amplitude $\epsilon_{0}$. The dependence on area ratio $A_{0} / A$ observed with $\omega_{0}=\pi$ (more specifically, a line drawn through the data for the lowest amplitude motion) is also indicated in figure 18. It is seen that a solid end or a small orifice, and also an open end or a large orifice, are more ideal reflectors at $\omega_{0}=\pi$ than at $\omega_{0}=\frac{1}{2} \pi$. By ideal we mean that the reflexion coefficient is closer to +1 or -1 , respectively. The reason for this remarkable result is not known, though it might be hypothesized that differences between the wave forms observed at $\omega_{0}=\pi$ and at $\omega_{0}=\frac{1}{2} \pi$ must be important. It is likely 

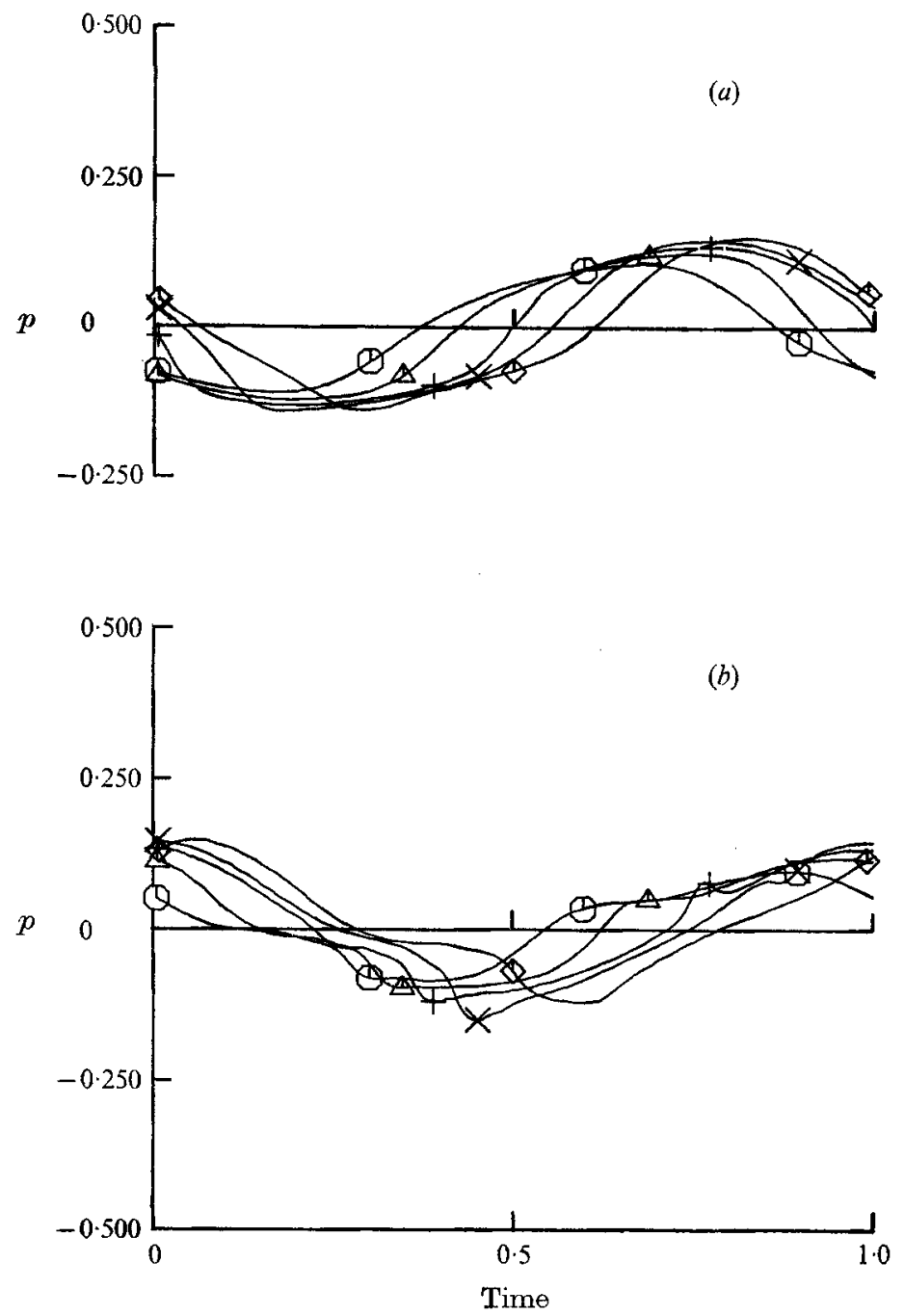

Frgune 16. Normalized pressure wave form in an open tube terminated with a, $5 \cdot 1 \mathrm{~cm}$ diameter orifice. (a) Pressure at piston end. (b) Pressure at open end. $\omega / \omega_{0}: 0,0.80 ; \Delta, 0.89$; ,$+ 0.98 ; \times, 1.05 ; \diamond, 1 \cdot 22$. File 94.09 .

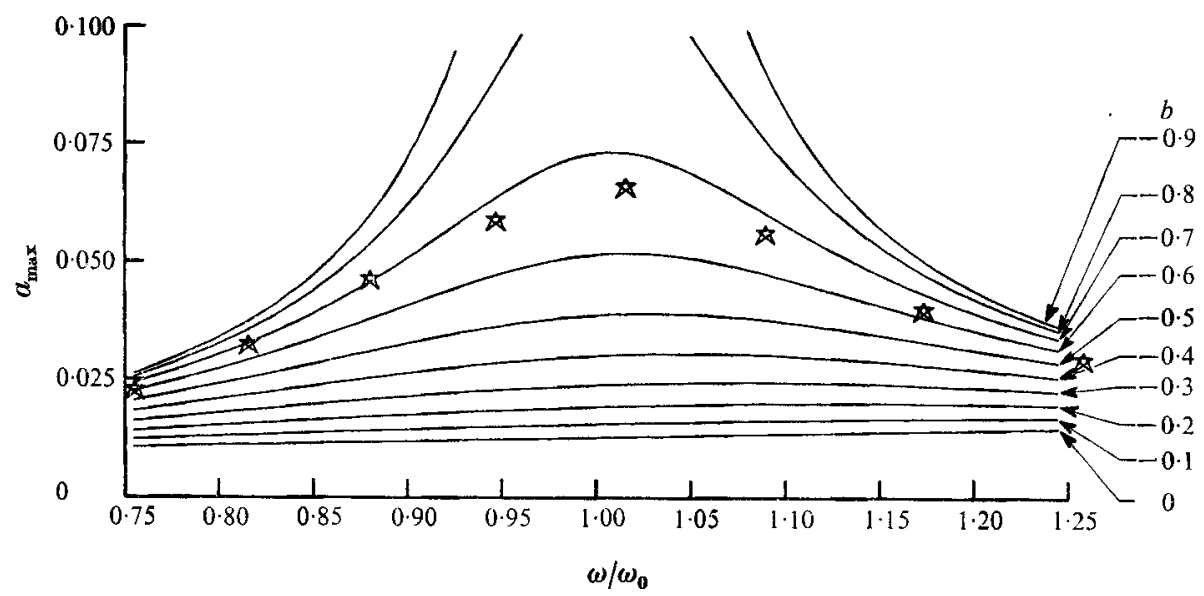

FIGURE 17. Comparison of experiment with linear theory for $\omega_{0}=\frac{1}{2} \pi$. Orifice diameter $=6.4 \mathrm{~cm} . \longrightarrow$, theory; , experiment. File 195.04 . 


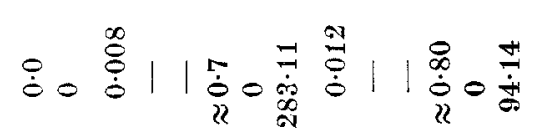

舟管号

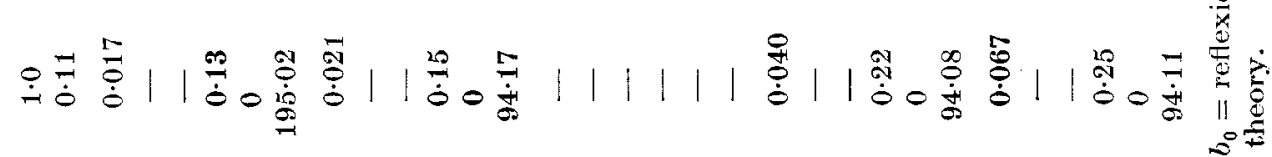

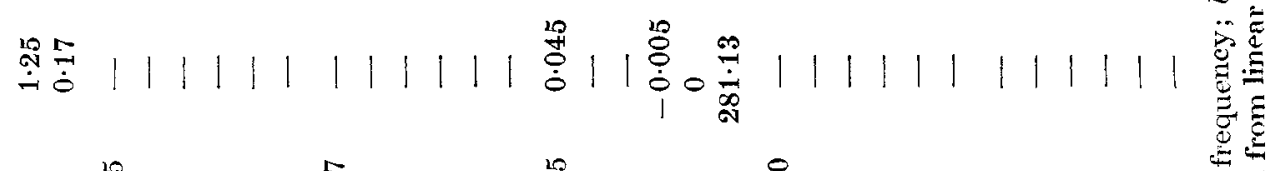

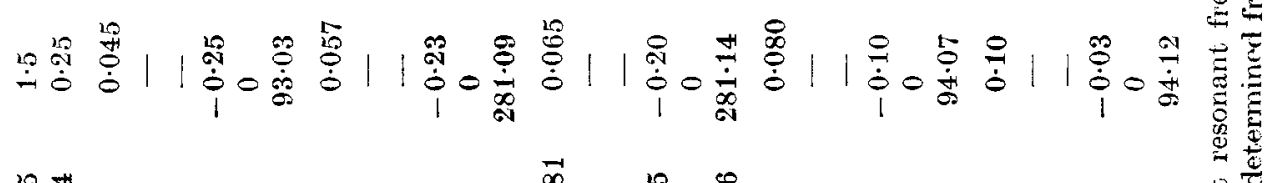

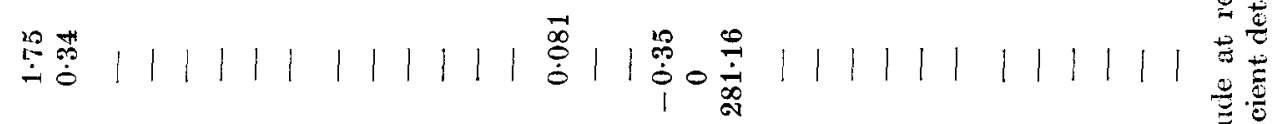

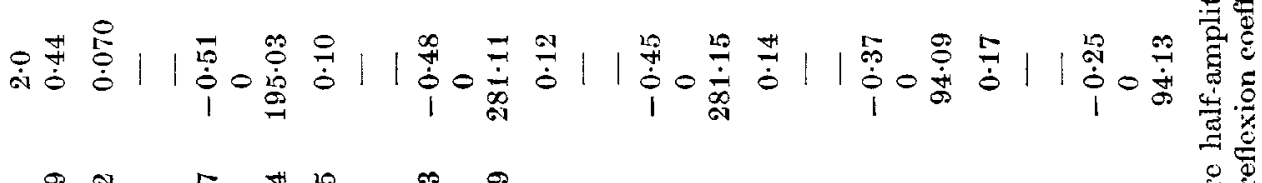

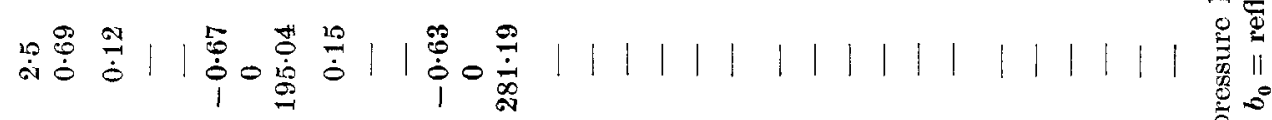

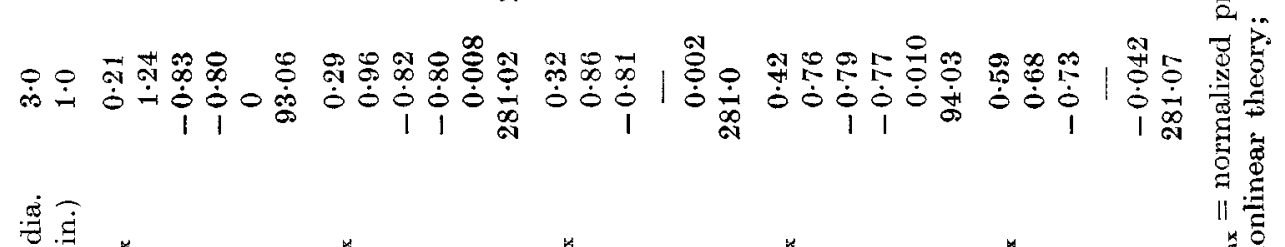

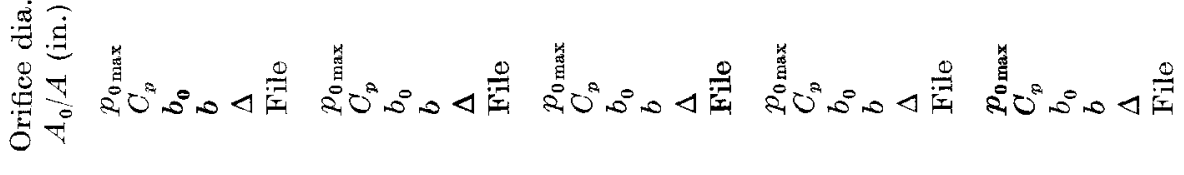

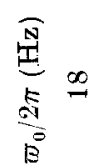

ล

$\infty$

8

$\infty$

$=\stackrel{10}{0}$

$\stackrel{\substack{i \\:}}{i}$

$\ddot{8}$

$\ddot{\circ}$

$\stackrel{9}{0}$

ह 닥

के

$\stackrel{\circ}{\text { ง }}$

5

$\stackrel{\circ}{\circ}$

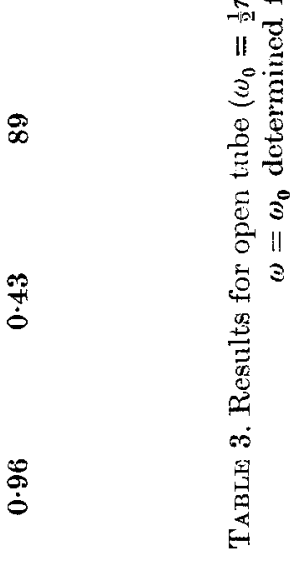




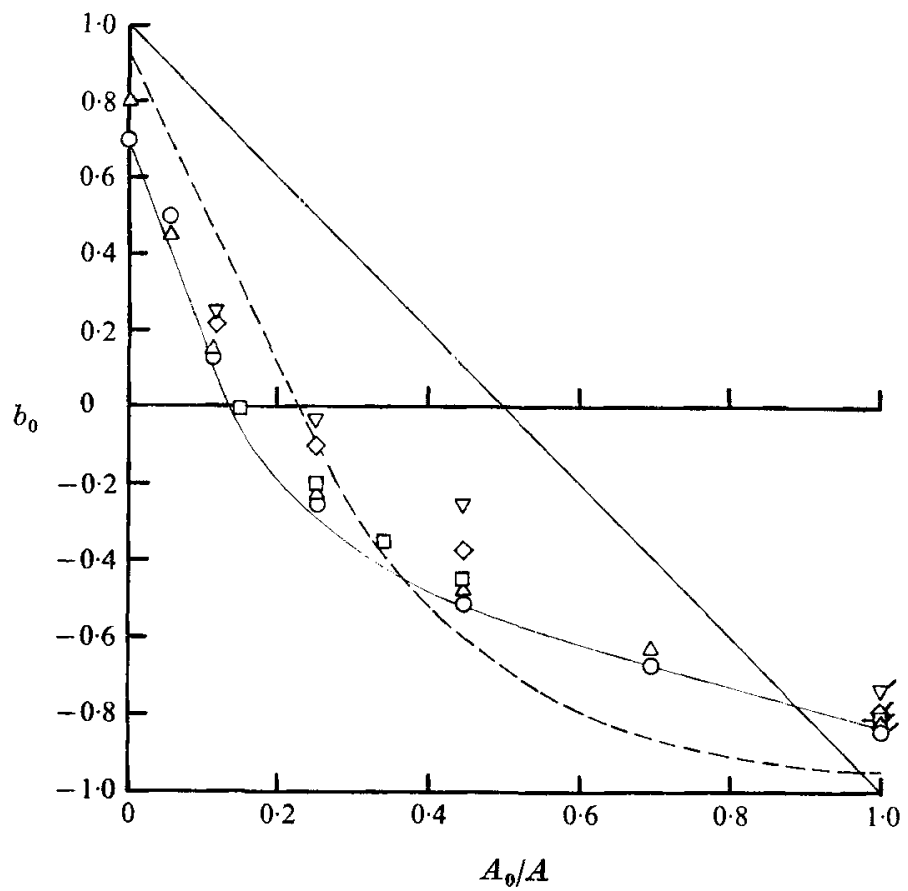

Figure 18. Reflexion coefficient at resonant frequency versus orifice area ratio for $\omega_{0}=\frac{1}{2} \pi$. Symbols with flags indicate data obtained from nonlinear theory; symbols without, data from linear theory. ---, closed-end results.

$\begin{array}{lllccc} & \nabla & \diamond & \square & \Delta & 0 \\ L(\mathrm{~m}) & 1 \cdot 0 & 1 \cdot 7 & 2 \cdot 6 & 3 \cdot 2 & 4 \cdot 7 \\ \epsilon_{0} & 0 \cdot 43 & 0 \cdot 36 & 0.31 & 0 \cdot 29 & 0 \cdot 25\end{array}$

that incorporation of a reflexion coefficient which depends on the instantaneous value of the orifice velocity rather than simply on its amplitude, as has been done for more conventional impedance tube measurements by Zorumski \& Parrott (1971), would reduce the observed discrepancy, but this is beyond the scope of the present work.

\section{Conclusions}

Measurements have been made of nonlinear oscillations in pipes. The effect on the motion of orifice plates inserted at the passive end of the tube has been studied.

It has been found that for olosed tubes when shock waves occur the effect of increasing orifice area is not only to weaken the shocks but also to thicken them. By adjusting both the effective length of the tube and the radiation coefficient very good agreement between theory and experiment may be obtained.

Oscillations at resonance in an open tube have remarkably large amplitude, owing to cancellation of second-order nonlinearities. Though the wave forms at the piston are continuous in our" experiments, in some cases compression waves steepen in less than one tube length to form shocks, and shock waves are emitted from the open end. The wave forms at the piston predicted by third-order theory 
are qualitatively similar to the observed wave forms, but consistently lag behind them by an observable amount.

By comparing the experimental results with nonlinear theory, wave reflexion coefficients of the orifice plates are determined at both closed-tube and opentube resonant frequencies. It is found that for both cases the reflexion coefficient of a given orifice increases with amplitude, in qualitative agreement with earlier studies of the nonlinear acoustic behaviour of orifices (Ingard \& Ising 1967). In fact, for oscillations at resonance in an open tube it is found that for fixed $\epsilon_{0}$ the radiation coefficient $b_{2}$ is proportional to the amplitude, so a wave pressure coefficient $C_{p}$ is used to measure the impedance of the open end. When the wave amplitude is changed by altering the normalized amplitude $l / L$ of the piston motion, $C_{p}$ varies somewhat; this is interpreted as a possible Reynolds number effect.

Quantitative differences are observed between the reflexion coefficient for oscillations at the open-tube and closed-tube resonant frequencies; reflexion from the end tends to be rather less ideal for $\omega_{0}=\frac{1}{2} \pi$ than for $\omega_{0}=\pi$ (i.e. the reflexion coefficient departs more significantly from -1 for an open end and from +1 for a solid end). The plane from which the waves effectively reflect tends to be outside the tube $(\Delta>0$, cf. table 3$)$ for $\omega_{0}=\frac{1}{2} \pi$. This is similar to the behaviour observed in classical acoustics. On the other hand, at $\omega_{0}=\pi$, in at least one case the effective plane of reflexion is inside the tube. It is possible that the dependence of the reflexion process on the wave form could be accounted for in the nonlinear theory by incorporating a reflexion coefficient which depends on the instantaneous amplitude, but the limitations of this approach are evidenced by the observation of Zorumski \& Parrott (1971) that even with such a modification it is necessary to introduce parameters dependent on the wave form, i.e. a rise time, to explain experimental results.

It has been shown that by use of a nonlinear theory the reflective properties of terminating elements can be defined and measured even when subjected to intense periodic pressure pulses. In acoustic terminology, pressure levels of from 140 to $190 \mathrm{db}$ have been observed in the present experiments. Future reports will describe measurements with more complicated terminating elements.

The author wishes to express his gratitude to his colleague $\mathrm{Mr} \mathrm{J}$. Jimenez for many helpful discussions. This work was supported in part by a grant from the National Aeronautics and Space Administration (NGR 05-002-220).

\section{REFERENCES}

Chester, W. 1964 J. Fluid Mech. 18, 44.

CruidKshank, D. B. 1972 J. Acoust. Soc. Am. 52, 1024.

Ingard, U. \& IsING, H. $1967 J$. Acoust. Soc. Am. 42, 6.

JiMENEZ, J. 1973 J. Fluid Mech. 59, 23.

LetraU, E. 1939 Dt. KraftfForsch. 39, 1.

RAYlexah, LoRD, 1945 The Theory of Sound. Dover.

Saenger, R. A. \& Hudson, G. E. 1960 J. Acoust. Soc. Am. 32, 961.

Sturtevant, B. 1970 Bull. Am. Phys. Soc. 15, 1546.

Temkin, S. 1968 Phys. Fluids, 11, 960.

Zorumski, W. E. \& Parrott, T. L. 1971 N.A.S.A. Tech. Note, D-6196. 